\title{
The BeppoSAX view of the galactic high-mass X-ray binary 4U 0114+65
}

\author{
N. Masetti ${ }^{1}$, M. Orlandini ${ }^{1}$, D. Dal Fiume ${ }^{2, \star}$, S. Del Sordo ${ }^{3}$, \\ L. Amati ${ }^{1}$, F. Frontera ${ }^{1,4}$, E. Palazzi ${ }^{1}$, and A. Santangelo ${ }^{5}$ \\ ${ }^{1}$ Istituto di Astrofisica Spaziale e Fisica Cosmica di Bologna, INAF, via Gobetti 101, 40129 Bologna, Italy (formerly IASF/CNR, Bologna) \\ e-mail: masetti@bo.iasf.cnr.it \\ 2 Istituto Tecnologie e Studio sulla Radiazione Extraterrestre, CNR, via Gobetti 101, 40129 Bologna, Italy \\ 3 Istituto di Astrofisica Spaziale e Fisica Cosmica di Palermo, INAF, via La Malfa 153, 90146 Palermo, Italy (formerly IASF/CNR, Palermo) \\ 4 Dipartimento di Fisica, Università di Ferrara, via Paradiso 12, 44100 Ferrara, Italy \\ 5 Institut für Astronomie und Astrophysik, Eberhard Karls Universität Tübingen, Sand 1, 72076 Tübingen, Germany
}

Received 17 June 2005 / Accepted 6 September 2005

\section{ABSTRACT}

A pointed observation of the galactic high-mass X-ray binary $4 \mathrm{U} 0114+65$ was carried out with BeppoSAX to compare the X-ray spectral and timing characteristics observed by this satellite over the broadest range of energies thus far $(1.5-100 \mathrm{keV})$ with the information previously obtained with other spacecraft. The light curve of $4 \mathrm{U} 0114+65$ shows a large flare at the beginning of the BeppoSAX pointing and no significant hardness evolution either during the flare or in the low state occurring after the flare itself. The modulation at $\sim 2.7 \mathrm{~h}$, attributed to the accreting neutron star (NS) spin periodicity, is not significantly detected in our data, although fluctuations with timescales of $\sim 3 \mathrm{~h}$ can be seen in the 2-10 keV light curve. Shorter modulations down to timescales of minutes are also found and interpreted as due to accretion of matter onto the NS. The flaring and the low state spectra of 4U 0114+65 can be equally well fitted either with a power law modulated by a high-energy exponential cutoff or with a Comptonization model. During the low state the presence, although tentative, of a thermal component (with $k T \sim 0.3 \mathrm{keV}$ ) at low energies, possibly produced by an ionized plasma cloud around the NS, cannot be excluded. Contrary to previous claims, a cyclotron resonant feature in absorption at $\sim 22 \mathrm{keV}$ was not detected in the BeppoSAX spectroscopic data, whereas evidence for a Fe emission line around $6.4 \mathrm{keV}$ is found only during the low state emission. Using all of the above information, a scenario for the system in which the NS is embedded in, and accreting from, a low angular momentum gas cloud is envisaged.

Key words. stars: binaries: close - X-rays: binaries - stars: neutron - stars: individual: 4U 0114+65 - accretion, accretion disks

\section{Introduction}

Persistently emitting High-Mass X-ray Binaries (HMXBs) are gravitationally bound systems composed of a early-type star losing matter, generally via stellar wind, in favour of a compact object, a neutron star (NS) or possibly a black hole. This accretion phenomenon induces high-energy emission from the accreting flow and, in the case of a NS as the accretor, from the surface of the compact object. This kind of HMXB has X-ray luminosities of the order of at least $10^{35} \mathrm{erg} \mathrm{s}^{-1}$ in the $2-10 \mathrm{keV}$ range. Also, if the accreting object is a magnetized NS, the accretion flow is channeled onto the magnetic polar caps and the $\mathrm{X}$-ray emission can appear as pulsed, modulated by the NS spin period (see White et al. 1995 for a review).

The hard X-ray source $4 \mathrm{U} 0114+65$ belongs to this group of X-ray binaries. It was discovered by the $S A S-3$ satellite

\footnotetext{
* Deceased.
}

(Dower et al. 1978) and extensively observed in the X-ray band by several spacecraft.

The lightcurve of the object appears quite variable in $\mathrm{X}$-rays, showing flares lasting a few hours, occurring on daily timescales and that are 15-20 times brighter than the persistent low-level emission (Yamauchi et al. 1990; Apparao et al. 1991). Shorter-term flickering on timescales of minutes was also observed (Koenigsberger et al. 1983). Finley et al. (1992), by analyzing archival EXOSAT and ROSAT data, showed the existence of a very stable 2.78 -h periodicity in the X-ray light curve of $4 \mathrm{U} 0114+65$; this was more recently confirmed by Corbet et al. (1999) and Hall et al. (2000) by using RXTE ASM and PCA data, respectively. This was interpreted by these authors in terms of the the spin period of a slowly rotating NS. Farrell et al. (2005) moreover reported the presence of a superorbital periodicity of 30.7 days in this system, again by the analysis of $R X T E / \mathrm{ASM}$ data. This may suggest the presence of a precessing accretion disk around the NS. 
X-ray spectroscopy (Yamauchi et al. 1990; Apparao et al. 1991; Hall et al. 2000) shows that the source spectrum is satisfactorily fitted with the "classical" phenomenological model for accreting NSs in HMXBs (White et al. 1983), i.e. a power law modified at high energies by an exponential cutoff. In the case of $4 \mathrm{U} 0114+65$, typical spectral parameter values are found to be $\Gamma \sim 1, E_{\text {cut }} \sim 8 \mathrm{keV}$ and $E_{\text {fold }} \sim 20 \mathrm{keV}$ for the photon index, the cutoff and folding energies. A neutral iron emission line at $6.4 \mathrm{keV}$ was also sometimes detected, preferentially during the persistent low state emission.

Bonning \& Falanga (2005) observed this source with INTEGRAL and again found the $2.7-\mathrm{h}$ spin modulation up to an energy of $80 \mathrm{keV}$. The $5-100 \mathrm{keV}$ INTEGRAL spectrum was consistent with the findings, illustrated above, of previous highenergy observations. They also measured a NS spin-up trend $\dot{P}=-8.9 \times 10^{-7} \mathrm{~s} \mathrm{~s}^{-1}$ over $\sim 8$ years. Moreover, these authors tentatively identified a Cyclotron Resonant Feature (CRF) at $\sim 22 \mathrm{keV}$ in the $\mathrm{X}$-ray spectrum of the source, implying a magnetic field of $2.5 \times 10^{12} \mathrm{G}$ for the accreting NS.

This evidence can be interpreted as due to accretion onto a NS from a wind coming from an early-type star. Indeed, $4 \mathrm{U} 0114+65$ was associated with its optical counterpart, the 11th magnitude star V662 Cas (also known as LSI $+65^{\circ} 010$ ) by Margon \& Bradt (1977). Subsequently, Crampton et al. (1985) spectroscopically determined the orbital period of the system as $P_{\text {orb }}=11$ d 59 ; this modulation was also found later in the RXTE/ASM X-ray data by Corbet et al. (1999). Reig et al. (1996) performed a thorough study of the optical properties of the companion star and found it is a blue supergiant of spectral type B1Ia located at $7.0 \pm 3.6 \mathrm{kpc}$ from earth, though Koenigsberger et al. (2003) note that it could be located closer. This star is in a basically circular orbit (Crampton et al. 1985; Koenigsberger et al. 2003) and may induce X-ray eclipses (Hall et al. 2000).

The above optical information conclusively placed $4 \mathrm{U} 0114+65$ among wind-accreting supergiant HMXBs in terms of spectral type of the companion and of X-ray luminosity budget, the latter being between $10^{35}$ and $10^{36} \mathrm{erg} \mathrm{s}^{-1}$ in the $2-10 \mathrm{keV}$ range; indeed, previous distance estimates ( $d \sim 2$ kpc; e.g., Aab et al. 1983) led to a $2-10 \mathrm{keV}$ luminosity of about ten times lower, erroneously implying that this source was a low-luminosity HMXB.

No radio emission was detected from this source in the $5 \mathrm{GHz}$ band down to a level of $1.2 \mathrm{mJy}$ (Nelson \& Spencer 1988).

We observed 4U 0114+65 with BeppoSAX (Boella et al. 1997a) within the framework of our program of pointed observations of HMXBs (Dal Fiume et al. 2000; Orlandini et al. 1998, 1999, 2000, 2004; Masetti et al. 2004) in order to describe its X-ray temporal and spectral behaviour over the wide range of energies $(0.1-300 \mathrm{keV})$ covered by this satellite, and to compare its X-ray characteristics with those of other objects belonging to this class.

Here we present the results of a pointed observation made with BeppoSAX on this system in January 1999. Thanks to the broadband spectroscopic capabilities of this satellite and to its high sensitivity, for the first time we were able to simultaneously explore the $1.5-100 \mathrm{keV}$ emission from this source. The
Table 1. Log of the BeppoSAX observation presented in this paper.

\begin{tabular}{ccccccc}
\hline \hline Start day & \multicolumn{2}{c}{ Start time } & Duration & \multicolumn{4}{c}{ On-source time (ks) } \\
& $(\mathrm{UT})$ & $(\mathrm{ks})$ & LECS & MECS & HPGSPC & PDS \\
\hline 1999 Jan. 26 & $21: 38: 42$ & 63.2 & 8.4 & 31.1 & 33.9 & 16.2 \\
\hline
\end{tabular}

paper is organized as follows: Sect. 2 describes the observations and the data analysis, in Sect. 3 the results showing the $\mathrm{X}$-ray spectral and timing behaviour of $4 \mathrm{U} 0114+65$ will be reported; in Sect. 4 a discussion is given.

\section{The BeppoSAX observation}

4U 0114+65 was observed between January 26 and 27, 1999 , for $\sim 63 \mathrm{ks}$, by the four coaligned Narrow-Field Instruments (NFIs) carried by BeppoSAX: the Low Energy Concentrator Spectrometer (LECS, 0.1-10 keV; Parmar et al. 1997), two Medium Energy Concentrator Spectrometers (MECS, 1.5-10 keV; Boella et al. 1997), the High-Pressure Gas Scintillation Proportional Counter (HPGSPC, 6-60 keV; Manzo et al. 1997) and the Phoswich Detection System (PDS, 15-300 keV; Frontera et al. 1997). The total duration of this BeppoSAX pointing along with the on-source exposure times for each NFI used are reported in Table 1.

The source was well detected by all four NFIs. Good NFI data were selected from intervals outside the South Atlantic Geomagnetic Anomaly when the elevation angle above the earth limb was $>5^{\circ}$, when the instrument functioning was nominal and, for LECS events, during spacecraft night time. The SAXDAS 2.0.0 data analysis package (Lammers 1997) was used for the extraction and the processing of LECS and MECS data. The PDS data reduction was performed using XAS version 2.1 (Chiappetti \& Dal Fiume 1997). LECS and MECS data were both reduced using an extraction radius of $4^{\prime}$ centered on the source position; before extraction, data from the two MECS units were merged. Background subtraction for the two imaging instruments was performed using standard library files. The background for the PDS data was evaluated from the fields observed during off-source pointing intervals, whereas that of the HPGSPC was computed through an Earth-occultation technique (Manzo et al. 1997).

Because $4 \mathrm{U} 0114+65$ is located near the Galactic plane and is not a particularly bright X-ray binary, we checked for possible effects induced by Galactic diffuse emission in the PDS data background evaluation. The PDS off-source fields for background evaluation were indeed at different Galactic latitudes $\left(b=-0.9\right.$ and $\left.b=+6^{\circ} .0\right)$ with respect to the source $(b=+2.6)$, so a possible gradient in the Galactic diffuse emission, if present, can be measured and eventually removed from the PDS data. However, the count rate difference between the two fields is $-0.06 \pm 0.07$ counts $\mathrm{s}^{-1}$, thus consistent with zero; moreover, this difference impacts on the background estimate by less than $0.3 \%$. Therefore we considered this effect negligible. 


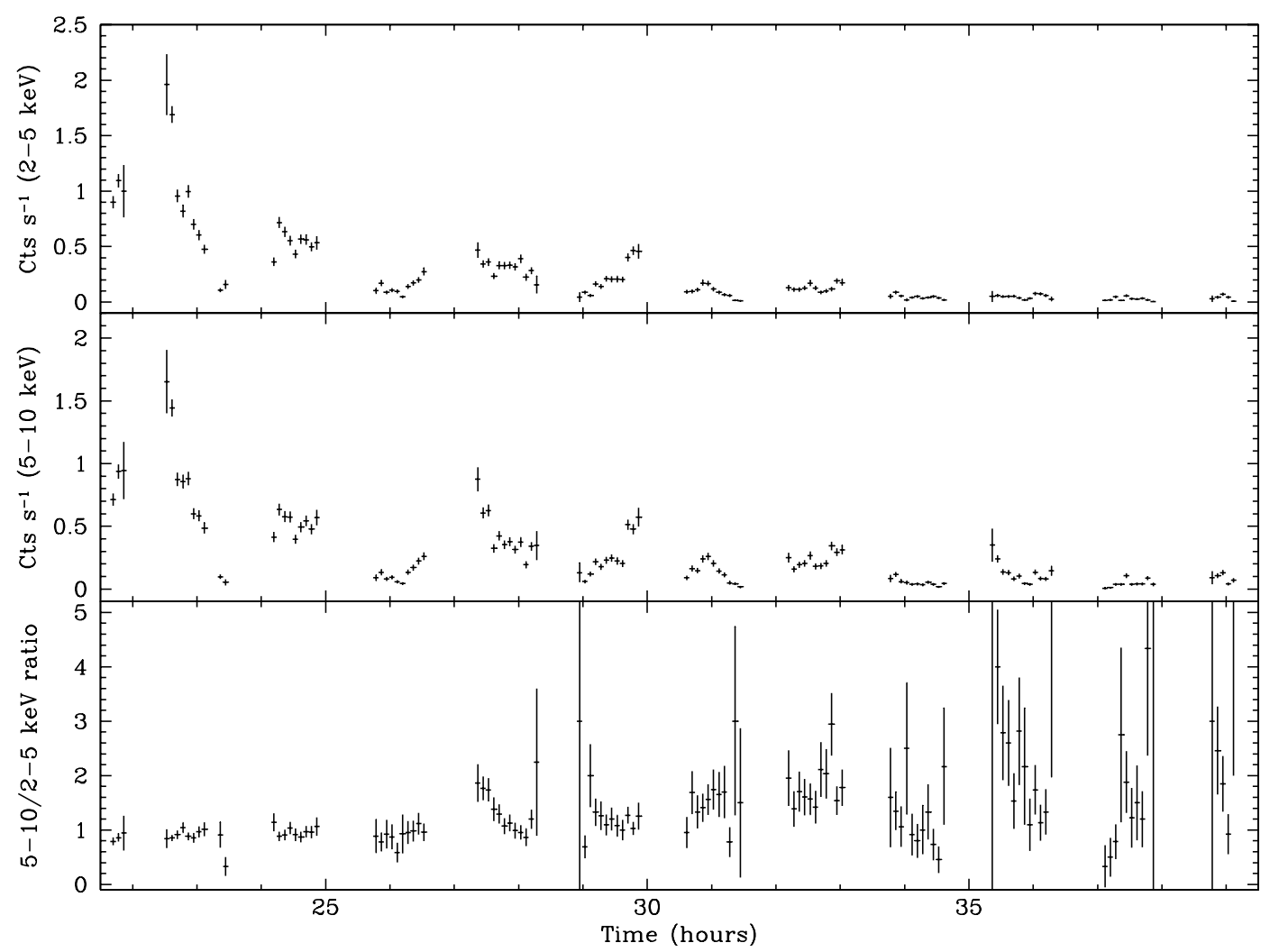

Fig. 1. Background-subtracted X-ray light curves of $4 \mathrm{U} 0114+65$ in the $2-5 \mathrm{keV}$ (upper panel), 5-10 keV (middle panel) bands, along with their ratio (lower panel) as observed during the BeppoSAX pointing. All curves are rebinned at $300 \mathrm{~s}$. A large flare during the first $3 \mathrm{~h}$ of the observation is apparent. Subsequent flickering activity down to timescales of minutes can be noticed. Time is measured from the start of the BeppoSAX observation, on January 26, 1999, at 21:38:42 UT (see also Table 1), and it is expressed in hours starting from 00:00 UT of January 26,1999 . Vertical error bars show $1 \sigma$ confidence level uncertainties for each bin.

\section{Results}

\subsection{Light curves}

We extracted the 2-5 and 5-10 keV light curves of $4 \mathrm{U} 0114+65$ from the BeppoSAX/MECS data. These background-subtracted MECS light curves rebinned at $300 \mathrm{~s}$, along with their ratio, are shown in Fig. 1. A large flare, similar to those reported by Apparao et al. (1991), was seen from 4U 0114+65 at the beginning of the BeppoSAX observation (Fig. 1). Its peak intensity is $\sim 20$ times the persistent low-level emission observed after the event. No significant hardness evolution was detected during the episode, although the $(5-10 \mathrm{keV}) /(2-5 \mathrm{keV})$ hardness ratio seems to increase starting $\sim 5 \mathrm{~h}$ after the beginning of the observation, just after the end of the flare (Fig. 1, lower panel). The light curves also show substantial random variability in the form of internal fluctuations lasting from a few hours to minutes (the so-called "X-ray flickering").

In order to see whether this erratic variability implied spectral changes depending on the source intensity, we computed a hardness-intensity ratio between the $5-10 \mathrm{keV}$ and the $2-5 \mathrm{keV}$ count rates and plotted it against the total $2-10 \mathrm{keV}$ count rate. The results are plotted in Fig. 2: although the figure hints to an inverse correlation between the two quantities, there is no significant dependence of the hardness ratio on the total intensity of $4 \mathrm{U} 0114+65$ in the $2-10 \mathrm{keV}$ range, as opposed to the inverse relation between X-ray hardness and intensity found by Apparao et al. (1991) from EXOSAT data.

Using the optical orbital ephemeris reported by Crampton et al. (1985) it is found that this large flare occurred just after periastron passage: in detail, we find that the flare occurred at phase $\phi \sim 0.05$ assuming the most likely case of circular orbits for the system (Koenigsberger et al. 2003).

\subsection{Timing properties}

Timing analysis on the $2-10 \mathrm{keV}$ data (where the $\mathrm{S} / \mathrm{N}$ ratio was highest) was performed with the FTOOLS v5.1 ${ }^{1}$ (Blackburn 1995) tasks powspec and efold, after having converted the event arrival times to the solar system barycentric frame.

The results do not reveal the presence of any kind of periodicity or quasiperiodic oscillations. We were not able to detect the $2.7 \mathrm{~h}$ NS spin periodicity in our analysis. The Power Spectral Density (PSD; Fig. 3) obtained with this analysis is characterized by red noise and shows no significant deviations from the $1 / f$-type distribution (where $f$ is the time frequency) typical of the "shot-noise" behaviour. This trend however flattens for frequencies lower than $f \approx 10^{-4} \mathrm{~Hz}$, which is of the order of magnitude of the $4 \mathrm{U} 0114+65$ NS spin frequency. Indeed, one can see from Fig. 1 that, at least in the first half

\footnotetext{
1 Available at:

http://heasarc.gsfc.nasa.gov/ftools/
} 


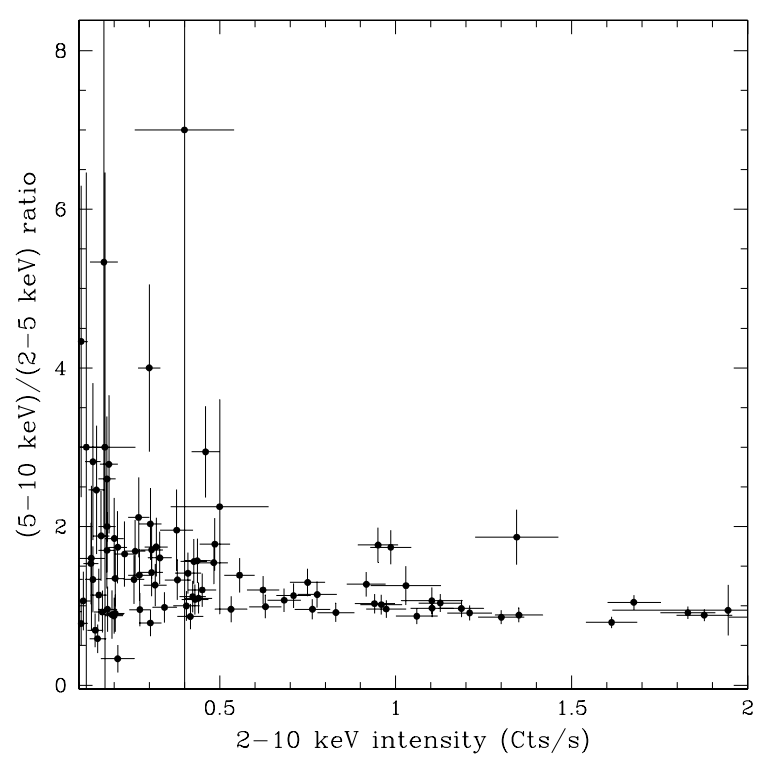

Fig. 2. Hardness-intensity diagram for the $2-10 \mathrm{keV}$ emission of $4 \mathrm{U} 0114+65$ during the BeppoSAX pointing. Although an hint of an inverse correlation between the $5-10 \mathrm{keV} / 2-5 \mathrm{keV}$ hardness ratio and the $2-10 \mathrm{keV}$ intensity is suggested by the plot, no statistically significant trend is present. Error bars show $1 \sigma$ confidence level uncertainties for each data point.

of the BeppoSAX observation, the presence of fluctuations on a timescale of $\sim 3 \mathrm{~h}$ is suggested. We stress that the non-detection of this modulation may be due to the relatively short duration of the observation ( $\sim 3$ pulse cycles if one considers the on-source time), to the sparse light curve sampling and to the presence of the large flare at the beginning of the pointing.

\subsection{Spectra}

To perform the spectral analysis, the NFI pulse-height spectra were rebinned to oversample by a factor of 3 the full width at half maximum $(F W H M)$ of the energy resolution and to have a minimum of 20 counts per bin, such that $\chi^{2}$ statistics could reliably be used. Data were then selected, for each NFI, in the energy intervals in which sufficient counts were detected from the source and for which the instrument response function was well determined. This led us to consider the spectral interval $1.5-4 \mathrm{keV}$ for the LECS, 1.6-10 keV for the MECS, 7-30 keV for the HPGSPC and 15-100 keV for the PDS. We then used the package XSPEC (Dorman \& Arnaud 2001) v11.3.1 to fit the resulting broad band energy spectrum.

We included in all fits described here an interstellar photoelectric absorption column, modeled using the Wisconsin cross sections as implemented in XSPEC (Morrison \& McCammon 1983) and with solar abundances as given by Anders \& Grevesse (1989).

When performing the spectral fits, normalization factors were applied to LECS, HPGSPC and PDS spectra following the cross-calibration tests between these instruments and the MECS (Fiore et al. 1999). These factors were constrained to be within the allowed ranges during the spectral fitting, with the possible exception of the HPGSPC one, with results slightly

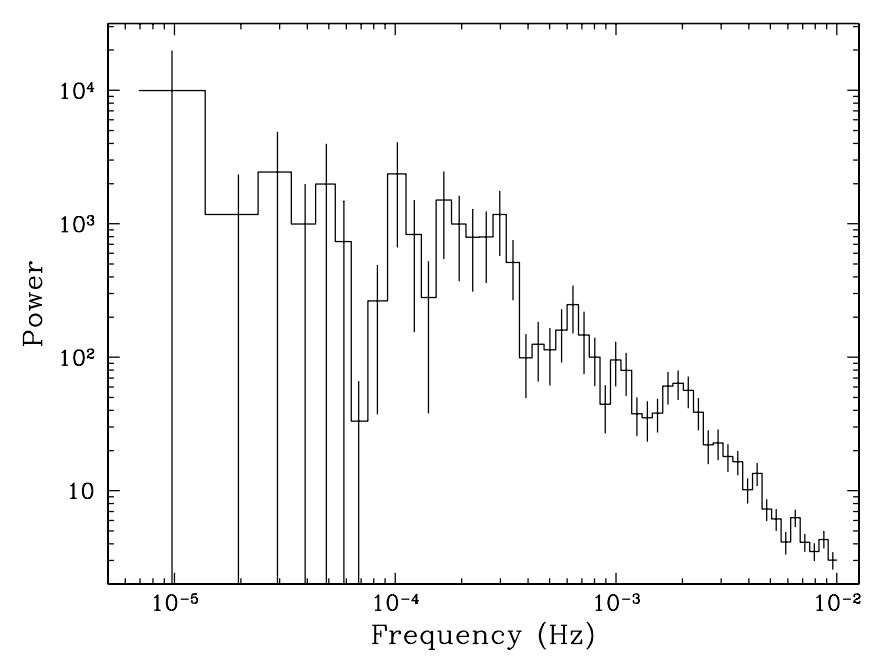

Fig. 3. 2-10 keV PSD of 4 U $0114+65$ obtained from the events registered by the two MECS units. The PSD is normalized according to the prescription by Leahy et al. (1983). No coherent oscillation or periodicity is found, whereas a shot-noise $1 / f$-type trend is apparent, flattening at frequencies below $f \approx 10^{-4} \mathrm{~Hz}$.

$(\sim 10 \%)$ higher than the expected range. This shift is however justified by the fact that the MECS and PDS data, in the ranges in which they overlap those of the HPGSPC, follow the same bin-to-bin variation pattern. We can moreover exclude the presence of a contaminating field source because (i) no catalogued sources with $2-10 \mathrm{keV}$ flux $>10^{-13} \mathrm{erg} \mathrm{cm}^{-2} \mathrm{~s}^{-1}$, i.e. $10^{3}$ times fainter than $4 \mathrm{U} 0114+65$, are present in the HPGSPC field of view $\left(1^{\circ} \times 1^{\circ} F W H M\right)$, (ii) the MECS and PDS instruments do not need such a higher shift for their intercalibration factor and, most importantly, (iii) no source other than 4U 0114+65 is detected in the field imaged by the MECS (which has a size comparable to that of the HPGSPC).

To explore possible spectral differences between the flaring and the low-level emission from $4 \mathrm{U} 0114+65$ which could be overlooked by the simple $2-10 \mathrm{keV}$ hardness-intensity ratio analysis of Fig. 2, we divided the observation into two parts using the data before and after 01:00 UT of January 27, 1999 (i.e., the 25th hour in the plot in Fig. 1). This allowed us to construct broad (1.5-100 keV) "flare" and "low-level" post-flare spectra for this source (reported in Fig. 3), therefore making the analysis of possible spectral changes described in Sect. 3.1 more complete. For these two time-resolved spectra Table 2 reports the models along with the corresponding parameters that best fit the data.

In the flaring spectrum, the "classical" phenomenological model of a power law (PL) plus a high-energy exponential cutoff (HIGHECUTPL; White et al. 1983) provides an acceptable fit to the data, with best-fit parameters which are similar to, but more accurately determined than those found e.g. by Bonning \& Falanga (2005) on an INTEGRAL X-ray spectrum with similar broadband coverage. An equivalently good fit (see Table 2) was also achieved using a PL with a simple cutoff (CUTOFFPL), in the form $N(E) \propto E^{-\Gamma} \cdot \mathrm{e}^{-E / E_{\text {cut }}}$.

Turning to a more physical description, as suggested by recent observational (e.g., Masetti et al. 2004; Torrejón et al. 2004) and theoretical (Becker \& Wolff 2005) results, we tried 
Table 2. Best-fit spectral parameters for the flaring and low-level emission of $4 \mathrm{U} 0114+65$. In the leftmost column of the table, $N_{\mathrm{H}}$ is the neutral hydrogen column density, $\Gamma$ is the power law photon index, $E_{\text {cut }}$ is the cutoff energy, $E_{\text {fold }}$ is the exponential folding energy, $T_{0}$ is the temperature of the Comptonized seed photons, $T_{\mathrm{e}^{-}}$is the temperature of the Comptonizing electron plasma, $\tau$ is the optical depth of the Comptonizing plasma, and the $K$ s are the normalization constants of each model. Quoted errors are at the $90 \%$ confidence level for a single parameter. Luminosities, corrected for interstellar Galactic absorption, are computed assuming a distance $d=7.0 \mathrm{kpc}$ and using the HIGHECUTPL model as reference; no significant differences are found if other models are used.

\begin{tabular}{lrr}
\hline \hline Model + parameters & \multicolumn{1}{c}{ Flare } & \multicolumn{1}{c}{ Low-level } \\
\hline CUTOFFPL: & & \\
$N_{\mathrm{H}}^{a}$ & $8.9_{-0.7}^{+0.8}$ & $17.6_{-1.7}^{+1.6}$ \\
$\Gamma$ & $0.86 \pm 0.15$ & $0.92_{-0.23}^{+0.19}$ \\
$E_{\mathrm{cut}}(\mathrm{keV})$ & $15_{-2}^{+3}$ & $18_{-4}^{+5}$ \\
$K_{\mathrm{PL}}\left(\times 10^{-2}\right)$ & $2.2_{-0.4}^{+0.6}$ & $0.7_{-0.2}^{+0.3}$ \\
$\chi^{2} /$ d.o.f. & $201.7 / 199$ & $273.2 / 194$
\end{tabular}

$\begin{array}{lrr}\text { HIGHECUTPL: } & & \\ N_{\mathrm{H}}^{a} & 9.7_{-0.9}^{+0.7} & 15.4_{-1.7}^{+2.0} \\ \Gamma & 1.33_{-0.16}^{+0.09} & 0.9 \pm 0.2 \\ E_{\text {cut }}(\mathrm{keV}) & 12_{-3}^{+2} & 6.0_{-0.7}^{+0.9} \\ E_{\text {fold }}(\mathrm{keV}) & 21_{-3}^{+4} & 17_{-3}^{+5} \\ K_{\mathrm{PL}}\left(\times 10^{-2}\right) & 3.4_{-0.6}^{+0.7} & 0.43_{-0.15}^{+0.25} \\ \chi^{2} / \text { d.o.f. } & 199.0 / 198 & 262.7 / 193\end{array}$

$\begin{array}{lrr}\text { CомРTT: } & & \\ N_{\mathrm{H}}^{a} & 5.5_{-0.7}^{+0.9} & 11.7_{-1.5}^{+1.7} \\ T_{0}(\mathrm{keV}) & 1.31_{-0.14}^{+0.12} & 1.51 \pm 0.15 \\ T_{\mathrm{e}^{-}}(\mathrm{keV}) & 7.8_{-1.1}^{+1.5} & 9.7_{-1.6}^{+2.4} \\ \tau & 4.1_{-0.6}^{+0.7} & 3.5 \pm 0.6 \\ K_{\text {CompTT }}\left(\times 10^{-3}\right) & 6.1_{-1.1}^{+1.2} & 1.4 \pm 0.3 \\ \chi^{2} \text { d.o.f. } & 212.7 / 198 & 261.9 / 192\end{array}$

\begin{tabular}{lrr} 
COMPST: & & \\
$N_{\mathrm{H}}^{a}$ & $10.7 \pm 0.7$ & $20.9 \pm 1.5$ \\
$T_{0}(\mathrm{keV})$ & $6.1_{-0.5}^{+0.6}$ & $7.5_{-1.0}^{+1.3}$ \\
$\tau$ & $10.4 \pm 0.9$ & $8.8_{-1.1}^{+1.3}$ \\
$K_{\mathrm{CompST}}\left(\times 10^{-2}\right)$ & $5.2_{-0.7}^{+0.8}$ & $1.9_{-0.3}^{+0.4}$ \\
$\chi^{2} /$ d.o.f. & $229.9 / 199$ & $290.0 / 194$ \\
\hline$L_{2-10 \mathrm{keV}}^{b}$ & 13.5 & 3.7 \\
$L_{10-100 \mathrm{keV}}^{b}$ & 24.1 & 7.9 \\
\hline
\end{tabular}

${ }^{a}$ In units of $10^{22} \mathrm{~cm}^{-2}$.

${ }^{b}$ In units of $10^{35} \mathrm{erg} \mathrm{s}^{-1}$.

a Comptonization description of the source flare spectrum via the model by Titarchuk (1994; COMPTT in XSPEC) and found that it also provided an acceptable description, with bestfit parameters reported in Table 2 . We found no statistically significant difference in the fit when using different geometries for the Comptonizing cloud. We therefore decided to assume the (default) spherical geometry. Also, using the simpler, non-relativistic modelization by Sunyaev \& Titarchuk (1980; COMPST in XSPEC) for the Comptonization, we obtained acceptable but nevertheless worse fits (see Table 2); in particular, this description systematically underestimates the data of the flare spectrum above $40 \mathrm{keV}$.

All of these models also describe the low-level spectrum of 4U 0114+65 above $3 \mathrm{keV}$ acceptably well. However, as one can see in Fig. 4, below this energy an apparent soft X-ray excess is found despite the high value of $N_{\mathrm{H}}$, with the overall $1.5-100 \mathrm{keV}$ fit providing a $\chi^{2} /$ d.o.f. $~ 1.3-1.4$ (where "d.o.f." stands for "degrees-of-freedom") independently of the chosen model among those listed in Table 2 . We tried to fit this excess with a thermal component, and found that the fit is marginally improved (to a reduced $\chi^{2} \sim 1.2$, which corresponds to a $90 \%$ confidence level according to the $F$-test; e.g., Press et al. 1992) using either a blackbody (BB) with $k T_{\text {th }} \sim 0.3 \mathrm{keV}$ and radius $R_{\text {th }} \sim 200 \mathrm{~km}$, or with a disk-BB model (DBB; Mitsuda et al. 1984) with similar temperature and radius, or with a hot diffuse thermal plasma (MEKAL model in XSPEC; Mewe et al. 1985), again with a similar temperature as of the BB model. In the case in which this thermal component is added to the Comptonization, an acceptable fit $\left(\chi^{2} /\right.$ d.o.f. $\left.=237.6 / 191\right)$ is also obtained if one forces the thermal emission to be the source of the Comptonization seed photons by imposing $k T_{\text {th }} \equiv k T_{0}$. The value of this parameter is again $\sim 0.3 \mathrm{keV}$, whereas those of the Comptonization do not differ from those reported in Table 2.

In Table 2 the unabsorbed luminosities of the source in the two brightness states and in the 2-10 and 10-100 keV ranges are also reported, assuming a distance to $4 \mathrm{U} 0114+65$ of $7.0 \mathrm{kpc}$ (Reig et al. 1996). To compute them, the best-fit PL plus high energy exponential cutoff (i.e., HIGHECUTPL) modeling described above as the reference spectral modelization of the source was used. No substantial differences in these luminosity values are found if one uses the PL plus simple cutoff or the Comptonization best-fit models.

Finally, we searched for the presence of an iron emission line around $6.4 \mathrm{keV}$ (modeled as a Gaussian) and of a CRF at $\sim 22 \mathrm{keV}$ (using the CYCLABS multiplicative model in XSPEC; Mihara et al. 1990; Makishima et al. 1990). From our best-fit descriptions there appears to be no need to include either of the two spectral features mentioned above in the 4U 0114+65 flaring state. Instead, concerning the low-level state, while we again did not find any evidence for a CRF, a broad (FWHM $1 \mathrm{keV}$ ) $\mathrm{Fe}$ emission is required with a probability of improvement by chance of $5.8 \times 10^{-5}$.

For each of the two parts of the BeppoSAX observation we thus computed the value (when applicable) or the $90 \%$ confidence level upper limits to the equivalent width $(E W)$ of a Fe emission at $6.4 \mathrm{keV}$ (assuming different line $F W H M$ s) and to the depth of a CRF at $22 \mathrm{keV}$ (assuming a line width of $10 \mathrm{keV}$, as found by Bonning \& Falanga (2005) and which is typical of magnetic NSs in HMXBs; e.g. Orlandini \& Dal Fiume 2001). The results, reported in Table 3, are independent of the chosen best-fit spectral description.

However, from a visual inspection of the right panel of Fig. 4, there is a hint of a feature around $20 \mathrm{keV}$ in the low-level 

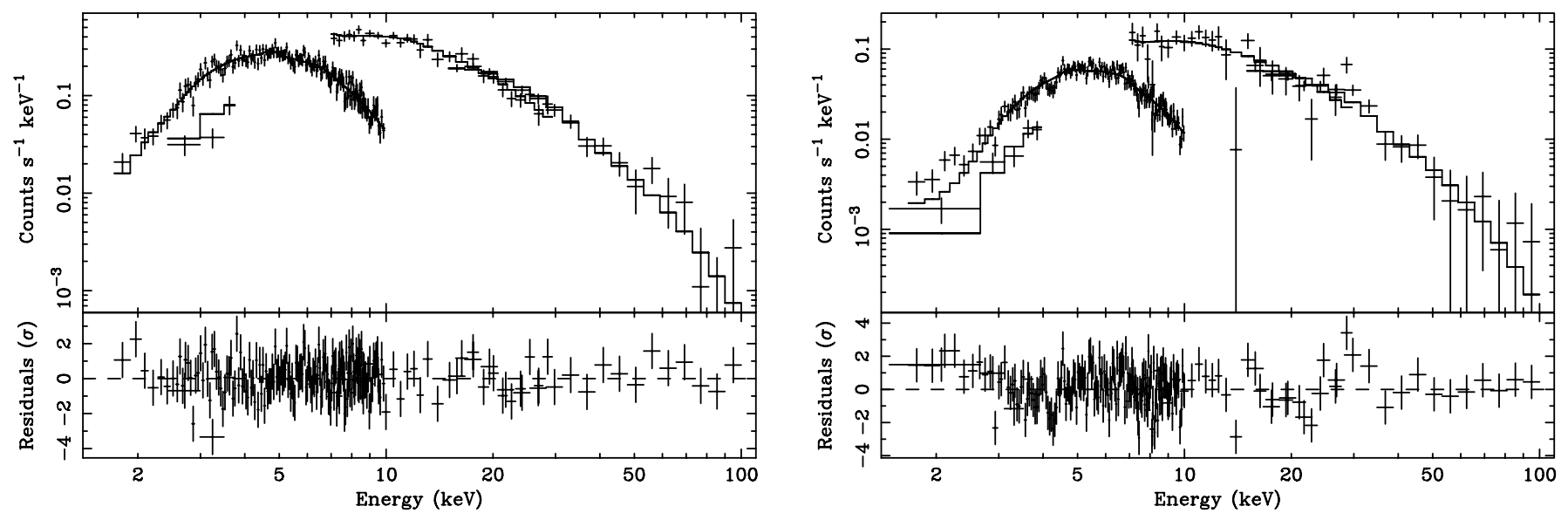

Fig. 4. Left panel: $1.5-100 \mathrm{keV}$ X-ray spectrum of $4 \mathrm{U}$ 0114+65 obtained with the BeppoSAX NFIs during the flare reported in Fig. 1 and fitted with a photoelectrically absorbed PL modified with a high energy exponential cutoff (HIGHECUTPL model of Table 2). The best-fit model is shown as a continuous line. Right panel: 1.5-100 X-ray spectrum of 4U 0114+65 obtained with the BeppoSAX NFIs during the low-level state following the flare reported in Fig. 1. The best-fit model is the same as in the left panel. Note the hint of a soft X-ray excess below $3 \mathrm{keV}$. For both panels, the residuals between data and model are shown in units of $\sigma$, and the differences in counts $\mathrm{s}^{-1} \mathrm{keV}^{-1}$ among the various instruments are due to different effective detector areas.

Table 3. Values and $90 \%$ confidence level upper limits to the $E W$ (in $\mathrm{eV}$ ) of the $6.4 \mathrm{keV} \mathrm{Fe}$ emission line, assuming three different fixed line $F W H M$ s, and to the CRF depth assuming an energy $E_{\mathrm{CRF}}=$ $22 \mathrm{keV}$ and a width of $10 \mathrm{keV}$.

\begin{tabular}{lrrrr}
\hline \hline Observation & \multicolumn{3}{c}{ Fe line $E W(\mathrm{eV})$} & CRF depth \\
& $0.1 \mathrm{keV}$ & $0.5 \mathrm{keV}$ & $1 \mathrm{keV}$ & \\
\hline Flare & $<61$ & $<93$ & $<141$ & $<0.3$ \\
Low state & $80 \pm 50$ & $250_{-90}^{+100}$ & $580 \pm 200$ & $<0.3$ \\
\hline
\end{tabular}

spectrum of $4 \mathrm{U} 0114+65$. In order to check whether the feature is real or due to a non-perfect modeling of the continuum we performed a Crab ratio of the PDS spectra for both brightness states. This technique was used to successfully pinpoint CRFs in numerous X-ray binary pulsars (see, e.g., Orlandini et al. 1998), and consists of obtaining the ratio between the count rate spectrum of the source and that of the featureless power law spectrum of the Crab. This ratio has the advantage of minimizing the effects due to detector response and calibration uncertainties. Any real feature present in the source spectrum can then be enhanced by multiplying the ratio by the functional form of the Crab spectrum (a power law with photon index $\Gamma=2.1$ ) and by dividing it by the functional form of the continuum adopted to fit the broad-band source spectrum, obtaining the so-called Normalized Crab Ratio (NCR; see Orlandini et al. 1998 for details). The NCR performed on both the flare and low-level 4U 0114+65 spectra does not show any incorrect modeling of the continuum and further excludes the presence of any absorption feature at $\sim 22 \mathrm{keV}$.

Also, if we force our low-level spectral data to be described by a spectrum like that of the best fit by Bonning \& Falanga (2005), we obtain a larger reduced $\chi^{2}(\sim 1.8)$, a larger HPGSPC intercalibration constant, and a wide fictituous emission bump between 20 and $50 \mathrm{keV}$, i.e., a trend in the fit residuals which is opposite with respect to that found by those authors. Because of this, we regard the description of our BeppoSAX low-level data using the best-fit results by Bonning \& Falanga (2005) as statistically not satisfactory.

\section{Discussion}

The BeppoSAX observation of $4 \mathrm{U} 0114+65$ presented in this paper allowed us to explore the X-ray behaviour of this source in the widest band thus far achieved (1.5-100 keV). Analysis of the X-ray light curves of the object shows that it underwent a large flare, with total duration $\sim 4 \mathrm{~h}$, at the beginning of the pointing. This was followed by a progressive return of the source to a low-level flux at an intensity about 20 times fainter than the peak of the flare. One can indeed see from Table 2 that the average flux level during the flare is more than $\sim 3$ times that of the average post-flare emission, going from $3.8 \times 10^{36} \mathrm{erg} \mathrm{s}^{-1}$ down to $1.2 \times 10^{36} \mathrm{erg} \mathrm{s}^{-1}$ in the $2-100 \mathrm{keV}$ band. Using the orbital ephemeris determined optically by Crampton et al. (1985) we found that this flare occurred just after periastron passage, at phase $\phi \sim 0.05$.

The modulation at $\sim 2.7 \mathrm{~h}$, attributed to the accreting NS spin periodicity, is not significantly detected in our data, although fluctuations with timescales of $\sim 3 \mathrm{~h}$ could be seen in the $2-10 \mathrm{keV}$ light curve, and the PSD of the source in this $\mathrm{X}$-ray range indicates a break around the corresponding temporal frequency $\left(\approx 10^{-4} \mathrm{~Hz}\right)$. This may be due to the relatively short duration of the observation ( $\sim 3$ pulse cycles if one considers the on-source time), to the sparse light curve sampling and to the presence of the large flare at the beginning of the pointing. X-ray flickering down to timescales of minutes was instead found, with its PSD being proportional to $1 / f$, where $f$ is the temporal frequency. These short-term variations in the X-ray flux may be due to the instability of the accretion process, or to the inhomogeneities in the accreting stellar wind (or both; see, e.g., Kaper et al. 1993). Thus, both of these longterm (flares) and short-term (flickering) "shot-noise" variabilities point to an explanation for this X-ray activity as due to 
random inhomogeneities in the accretion flow onto a compact object (e.g. van der Klis 1995).

The possible presence of spectral evolution was investigated through a $2-10 \mathrm{keV}$ hardness-intensity diagram and by accumulating the $1.5-100 \mathrm{keV}$ X-ray spectra of the flare and of the low-level post-flare emission. The hardness-intensity diagram does not decisively show appreciable color evolution in the 2-5 keV and 5-10 keV bands flux; on the other hand, the comparison of the flare and low-level spectra of $4 \mathrm{U}$ 0114+65 as observed by BeppoSAX shows that (leaving aside the obvious variation in the model normalization due to the change in the flux level) no substantial modifications in the spectral parameters are apparent, with the notable exception of the neutral hydrogen column density $N_{\mathrm{H}}$.

Indeed, its value nearly doubles at lower emissions independently of the chosen best-fit spectral modelization. This trend was already suggested by the EXOSAT, Ginga and RXTE data (Apparao et al. 1991; Yamauchi et al. 1990; Hall et al. 2000). However, when compared to those previous observations, we find a larger value for $N_{\mathrm{H}}$. This difference can be explained by the fact that the $N_{\mathrm{H}}$ measurement obtained with BeppoSAX is substantially more accurate thanks to the better sensitivity and spectral coverage at low energies afforded by the BeppoSAX LECS and MECS. Thus, in our opinion the $N_{\mathrm{H}}$ value obtained with BeppoSAX should be considered as the correct one.

We also note that the $N_{\mathrm{H}}$ values we found in both flare and low-level spectra are substantially higher than the Galactic one along the $4 \mathrm{U} 0114+65$ line of sight, which is $N_{\mathrm{H}}^{\mathrm{G}}=$ $0.76 \times 10^{22} \mathrm{~cm}^{-2}$ according to Dickey \& Lockman (1990). This fact is also confirmed by the optical $V$-band absorption estimate along the LSI $+65^{\circ} 010$ line of sight made by Reig et al. (1996): the value they obtain, $A_{\mathrm{V}}=3.84$, corresponds to a $N_{\mathrm{H}}=0.69 \times 10^{22} \mathrm{~cm}^{-2}$ using the empirical formula by Predehl \& Schmitt (1995). This is consistent with the Galactic $N_{\mathrm{H}}$ value and substantially smaller than the $N_{\mathrm{H}}$ we found from $\mathrm{X}$-ray spectral fits. This difference is very likely due to the fact that the stellar wind absorbs soft X-rays, but does not cause an increase in $E(B-V)$, and thus $A_{\mathrm{V}}$, because the wind does not contain dust (see, e.g., Cox et al. 2005). This fact further suggests the presence of a noticeable and variable amount of neutral gas local to the system and very close to the NS (possibly, the accretion stream itself).

A variation in the characteristic energy $E_{\text {cut }}$ and (to a lesser extent) in the values of $E_{\text {fold }}$ and $\Gamma$ of the HIGHECUTPL model is also found, with these parameters decreasing in value as the total flux decreases. This trend is opposite to what was found by Hall et al. (2000) in the RXTE data. This may at least partly be due to the wider spectral coverage afforded by BeppoSAX, which allowed a better sampling of the X-ray spectral states of 4U 0114+65.

Concerning the possible presence of a further component in the softer part of the spectrum (below $3 \mathrm{keV}$ ), we note that Hickox et al. (2004) found that a soft excess, usually modeled using a $\mathrm{BB}$ with $k T_{\mathrm{BB}} \sim 0.1 \mathrm{keV}$, is ubiquitous in X-ray pulsars (XRPs) and, in particular, in HMXBs hosting pulsating NSs. According to them, XRPs with luminosities larger than $\sim 10^{38} \mathrm{erg} \mathrm{s}^{-1}$ are produced by reprocessing of hard X-rays emitted by the NS by optically thick accreting material (e.g., an accretion disk), whereas in cases of XRPs with luminosities below $\sim 10^{36} \mathrm{erg} \mathrm{s}^{-1}$ this mechanism can be excluded and, most likely, the soft excess arises from different processes, namely emission from either a photoionized, or collisionally heated, diffuse gas or from the NS surface.

Given the size, exceeding the typical NS radius of a factor $\sim 20$, that we found for the thermal component responsible for the possible soft excess in $4 \mathrm{U} 0114+65$, we suggest that a cloud of diffuse plasma around the NS produces this excess.

The Fe emission, its variability and the fact that it is better seen during low emission levels were noted by Yamauchi et al. (1990) and Hall et al. (2000). This emission line may arise from the heated gas cloud depicted above as the possible cause of the soft X-ray excess and, as with the continuum emission from this cloud, is apparently better detected when $4 \mathrm{U} 0114+65$ is in the low state.

We did not find a CRF at $22 \mathrm{keV}$ in any of the two brightness states; this is at variance with the findings of Bonning \& Falanga (2005), who do not report any value for the line depth of their CRF detection. Therefore we are not able to confirm the estimate of the NS magnetic field strength made by these authors and, in turn, the magnetar origin proposed by Li \& van den Heuvel (1999) for the NS hosted in this system.

From visual inspection of the right panel of Fig. 4, there is a suggestion of a feature at $\sim 20 \mathrm{keV}$ in the low-level spectrum of 4 U $0114+65$. Using the NCR technique, we demonstrated in Sect. 3.3 that this feature is not due to an incorrect modeling of the X-ray spectral continuum, and thus we exclude the presence of any absorption feature at $\sim 22 \mathrm{keV}$ in our BeppoSAX data at both flux levels.

This same situation is observed for another X-ray binary pulsar: Vela $\mathrm{X}-1$. As pointed out by Orlandini \& Dal Fiume (2001) and Coburn et al. (2002), in the 15-30 keV energy interval there seems to occur a change of slope that is not well described in terms of actual spectral models, and that can give rise to residuals that can be misinterpreted as absorption features (see also the discussion on the X-ray binary pulsar OAO 1657-415 in Orlandini et al. 1999).

The above findings suggest the following scenario for $4 \mathrm{U} 0114+65$ : the accretion stream from the secondary star, in the form of a low angular momentum stellar wind as suggested by, e.g., Reig et al. (1996), is captured by the accreting NS and creates a plasma cloud around the accretor. This cloud thermally emits at a temperature $k T \sim 0.3 \mathrm{keV}$ and also produces an iron emission line. The thermal emission of the cloud is best seen at low hard X-ray flux levels, when this plasma cloud is denser, otherwise it is overwhelmed by the more intense underlying harder X-ray emission from the NS surface and/or from the NS-cloud boundary layer.

The total luminosity of the source is then driven by the density of the accreting cloud: when higher, it has the double effect of masking the inner harder emission and of producing a more intense softer thermal emission from its outer parts. Within this scenario, the presence of the superorbital period reported in X-rays by Farrell et al. (2005) cannot be explained by the presence of a precessing warped accretion disk; rather, it might be produced by the NS spin axis precession. 
Acknowledgements. This work has made use of the ASI Science Data Centre Archive at ESA/ESRIN, Frascati, of the NASA's Astrophysics Data System and of the SIMBAD database, operated at CDS, Strasbourg, France. BeppoSAX was a joint program of Italian (ASI) and Dutch (NIVR) space agencies. This research has been partially supported by ASI. We thank Antonino La Barbera for an independent check of the HPGSPC spectra presented in this paper, and the referee, Lex Kaper, for several useful comments which helped us to improve the paper.

\section{References}

Aab, O. É., Bychkova, L. V., \& Kopylov, I. M. 1983, Soviet Astron. Lett., 9, 285

Anders, E., \& Grevesse, N. 1989, Geochim. Cosmochim. Acta, 53, 197

Apparao, K. M. V., Bisht, P., \& Singh, P. 1991, ApJ, 371, 772

Becker, P. A., \& Wolff, M. T. 2005, ApJ, 630, 465

Blackburn, J. K. 1995, in Astronomical Data Analysis Software and Systems IV, ed. R. A. Shaw, H. E. Payne, \& J. J. E. Hayes, ASP Conf. Ser. (San Francisco: ASP), 77, 367

Boella, G., Butler, R. C., Perola, G. C., et al. 1997a, A\&AS, 122, 299

Boella, G., Chiappetti, L., Conti, G., et al. 1997b, A\&AS, 122, 327

Bonning, E. W., \& Falanga, M. 2005, A\&A, 436, L31

Chiappetti, L., \& Dal Fiume, D. 1997, The XAS Data Analysis System, in Proc. of the Fifth International Workshop on Data Analysis in Astronomy, ed. V. di Gesù, M. J. B. Duff, A. Heck, M. C. Maccarone, L. Scarsi, \& H.-U. Zimmermann (Singapore: World Scientific Pub.), 101

Coburn, W, Heindl, W. A., Rothschild, R. E., et al. 2002, ApJ, 580, 394

Corbet, R. H. D., Finley, J. P., \& Peele, A. G. 1999, ApJ, 511, 876

Cox, N. L. J., Kaper, L., Foing, B. H., \& Ehrenfreund, P. 2005, A\&A, 438, 187

Crampton, D., Hutchings, J. B., \& Cowley, A. P. 1985, ApJ, 299, 839

Dal Fiume, D., Orlandini, M., Del Sordo, S., et al. 2000, Adv. Space Res., 25, 399

Dickey, J. M., \& Lockman, F. J. 1990, ARA\&A, 28, 215

Dorman, B., \& Arnaud, K. A. 2001, Redesign and reimplementation of XSPEC, in Astronomical Data Analysis Software and Systems X, ed. F. R. Harnden Jr., F. A. Primini, \& H. E. Payne (San Francisco: ASP), ASP Conf. Ser., 238, 415

Dower, R. G., Bradt, H. V., Doxsey, R. E., et al. 1978, Nature, 273, 364

Farrell, S. A., Sood, R. K., \& O’Neill, P. M. 2005, MNRAS, submitted [arXiv: astro-ph/0502008]

Finley, J. P., Belloni, T., \& Cassinelli, J. P. 1992, A\&A, 262, L25

Fiore, F., Guainazzi, M., \& Grandi, P. 1999, Technical Report 1.2, BeppoSAX scientific data center, available online at: ftp://www.sdc.asi.it/pub/sax/doc/ software_docs/saxabc_v1.2.ps

Frontera, F., Costa, E., Dal Fiume, D., et al. 1997, A\&AS, 122, 357

Kaper, L., Hammerschlag-Hensberge, G., \& van Loon, J. T. 1993 , A\&A, 279, 485
Koenigsberger, G., Swank, J. H., Szymkowiak, A. E., \& White, N. E. 1983, ApJ, 268, 782

Koenigsberger, G., Canalizo, G., Arrieta, A., Richer, M. G., \& Georgiev, L. 2003, Rev. Mex. Astron. Astrofis., 39, 17

Hall, T. A., Finley, J. P., Corbet, R. H. D., \& Thomas, R. C. 2000, ApJ, 536,450

Hickox, R. C., Narayan, R., \& Kallman, T. R. 2004, ApJ, 614, 881

Lammers, U. 1997, The SAX/LECS Data Analysis System User Manual, SAX/LEDA/0010

Leahy, D. A., Darbro, W., Elsner, R. F., et al. 1983, ApJ, 266, 160

Li, X.-D., \& van den Heuvel, E. P. J. 1999, ApJ, 513, L45

Makishima, K., Mihara, T., Ishida, M., et al. 1990, PASJ, 365, L59

Manzo, G., Giarrusso, S., Santangelo, A., et al. 1997, A\&AS, 122, 341

Masetti, N., Dal Fiume, D., Amati, L., et al. 2004, A\&A, 423, 311

Mewe, R., Groenschild, E. H. B. M., \& van den Oord, G. H. J. 1985 A\&AS, 62,197

Mihara, T., Makishima, K., Ohashi, T., Sakao, T., \& Tashiro, M. 1990, Nature, 346, 250

Mitsuda, K., Inoue, H., Koyama, K., et al. 1984, PASJ, 36, 741

Morrison, R., \& McCammon, D. 1983, ApJ, 270, 119

Nelson, R. F., \& Spencer, R. E. 1988, MNRAS, 234, 1105

Orlandini, M., \& Dal Fiume, D. 2001, Hard X-ray tails and cyclotron features in X-ray pulsars, in X-ray astronomy, ed. N. E. White, G. Malaguti \& G. G. C. Palumbo, AIP Conf. Proc. (Melville, NY: AIP), 599, 283

Orlandini, M., Dal Fiume, D., Frontera, F., et al. 1998, A\&A, 332, 121

Orlandini, M., Dal Fiume, D., Del Sordo, S., et al. 1999, A\&A, 349, L9

Orlandini, M., Dal Fiume, D., Frontera, F., et al. 2000, Adv. Space Res., 25, 417

Orlandini, M., Bartolini, C., Campana, S., et al. 2004, X-ray/optical observations of A0535+26/HDE 245770 in quiescence, in II BeppoSAX Symposium "The Restless High-Energy Universe", ed. E. P. J. van den Heuvel, J. J. M. in 't Zand, \& R. A. M. J. Wijers, Nucl. Phys. B. Suppl. Ser., 132, 476

Parmar, A. N., Martin, D. D. E., Bavdaz, M., et al. 1997, A\&AS, 122, 309

Predehl, P., \& Schmitt, J. H. M. M. 1995, A\&A, 293, 889

Press, W. H., Teukolsky, S. A., Vetterling W. T., \& Flannery, B. P. 1992, Numerical Recipes (Cambridge: Cambridge Univ. Press)

Reig, P., Chakrabarty, D., Coe, M. J., et al. 1996, A\&A, 311, 879

Sunyaev, R. A., \& Titarchuk, L. 1980, A\&A, 86, 121

Titarchuk, L. 1994, ApJ, 434, 570

Torrejón, J. M., Kreykenbohm, I., Orr, A., Titarchuk, L., \& Negueruela, I. 2004, A\&A, 423, 301

van der Klis, M. 1995, in X-ray Binaries, ed. W. H. G. Lewin, J. van Paradijs, \& E. P. J. van den Heuvel (Cambridge: Cambridge Univ. Press), 252

Yamauchi, S., Asaoka, I., Kawada, M., Koyama, K., \& Tawara, Y. 1990, PASJ, 42, L53

White, N. E., Swank, J. H., \& Holt, S. S. 1983, ApJ, 270, 711

White, N. E., Nagase F., \& Parmar A. N. 1995, The properties of X-ray binaries, in X-ray Binaries, ed. W. H. G. Lewin, J. van Paradijs, \& E. P. J. van den Heuvel (Cambridge: Cambridge Univ. Press) 